\title{
Investigating photoproduction of scalar mesons at medium energies
}

\author{
M. L. L. da Silva ${ }^{1}$ and M. V. T. Machado ${ }^{2}$ \\ ${ }^{1}$ Instituto de Física e Matemática, Universidade Federal de Pelotas Caixa Postal 354, CEP 96010-090, Pelotas, Rio Grande do Sul, Brazil \\ ${ }^{2}$ High-Energy Physics Phenomenology Group, GFPAE IF-UFRGS Caixa Postal 15051, CEP 91501-970, Porto Alegre, \\ Rio Grande do Sul, Brazil \\ (Received 22 December 2011; published 24 July 2012)
}

\begin{abstract}
In this paper we study the photoproduction of scalar mesons in the intermediate energies, considering distinct mixing scenarios in the description of meson physical states. The differential and integrated total cross sections are computed for the cases of the mesons $a_{0}(980), f_{0}(1500)$, and $f_{0}(1710)$, focusing on the GlueX energy regime with photon energy $E_{\gamma}=9 \mathrm{GeV}$. Our results indicate that light-quark scalar meson photoproduction is well suited for studying hybrid mesons' structures.
\end{abstract}

DOI: $10.1103 /$ PhysRevC.86.015209

PACS number(s): 25.20.Lj, 12.38.-t, 13.60.Le, 14.40.-n

\section{INTRODUCTION}

For a long time, the scalar sector of mesons has been problematic and is still a subject of debate. The low-energy scalar states, for instance, the $f_{0}(980)$ and $a_{0}(980)\left(J^{P C}=0^{++}\right)$ states, have been considered conventional quark-antiquark mesons [1], tetraquarks [2], hadron molecules [3], glueballs, and hybrids $[4,5]$. In addition, for scalar mesons $f_{0}(1500)$ and $f_{0}(1710)$, there is not yet consensus on their status [6,7]. Such a confusing interpretation comes from the fact that despite the QCD providing a clear description of the strong interaction of partons (quarks and gluons) at high energies, the situation is complex in the low-energy regime. Namely, obtaining quantitative predictions from QCD at low energy, like the spectra of baryons and mesons, remains challenging and nowadays relies on numerical techniques of lattice QCD (LQCD). The current understanding of how quarks form mesons has evolved within QCD, and it is expected that a richer spectrum of mesons will take into account not only the quark degrees of freedom but also the gluonic ones. A common example is the glueball resonance with no quarks, which is expected to have quantum numbers that are not exotic and cannot be accommodated within quark-antiquark nonets [8]. This glueball state can mix with quark-antiquark states with the same quantum numbers. We also expect that excitations of the gluonic field from the quark binding could generate the so-called hybrid mesons, which are quark-antiquark states plus a one or more gluonic degrees of freedom. Thus, the exotic mesons provide the ideal laboratory for testing QCD in the confinement regime once they explicitly manifest the gluonic degrees of freedom [9].

The mesons $f_{0}(1500)$ and $f_{0}(1710)$ are considered good candidates for the scalar glueball $[6,7]$. However, in this mass region, the glueball state will mix strongly with nearby $q \bar{q}$ states $[10,11]$. In the lowest order, mixture of the scalar glueball $G$ and quarkonia states $n \bar{n}=(u \bar{u}+d \bar{d}) / \sqrt{2}$ and $s \bar{s}$ can be obtained as in Refs. [6,7]. The mixing is written in the following form: $\left|f_{0}(M)\right\rangle=c_{1}|n \bar{n}\rangle+c_{2}|s \bar{s}\rangle+c_{3}|G\rangle$, where the normalization condition is $\sum_{i=1}^{3} c_{i}^{2}=1$. In the literature the parameters have been adjusted to the observed resonances $f_{0}(1370), f_{0}(1500)$, and $f_{0}(1710)$ and are obtained from the mass of the glueball $n \bar{n}$ and $s \bar{s}$ states [7]. Much about the mixing parameters still remains unknown, and setting the parameters is very important to determine the structure of this resonances.

Despite an active experimental program, data supporting the existence of meson states having exotic quantum numbers are still sparse $[8,12]$. Concerning the scalar sector, the $f_{0}(1500)$ and $f_{0}(1710)$ are possibilities for the ninth member of the $S U(3)$ flavor nonet [13]. It is in general assumed that the surplus of isoscalar scalar quantities in the mass region $1300-1700 \mathrm{MeV}$ is due to the presence of a scalar glueball, which was supported by calculation on quenched LQCD [14]. Those mesons were viewed as mixed quarkantiquark and gluonium states. The interpretation has changed when considering unquenched LQCD calculations [15]. As an alternative the radiative transitions could offer a means of probing the structure of hadrons as the coupling to the charges and spins of the constituents reveals detailed information about the wave functions and could discriminate among models. Radiative decays of $f_{0}$ mesons to $\rho$ and $\omega$ have been shown to provide effective probes of their structure. If the $f_{0}$ mesons are mixed states, their radiative decays to vector mesons are strongly affected by the degree of mixing between the $q \bar{q}$ state and the glueball [16]. Following Refs. [16,17] and assuming that the $q \bar{q}$ contribution to the referred mesons is in the $1^{3} P_{0}$ nonet, the discrimination among different mixing possibilities is strong.

In this context, the photoproduction of exotic meson [17] is interesting for several reasons. Using arguments based on vector meson dominance (VDM), the photon can behave like an $S=1$ quark-antiquark system. In several models, such a system is more likely to couple to exotic quantum number hybrids. Recently, LQCD calculations have been performed to compute the radiative decay of charmonium and hybrid states [18], verifying a large radiative decay for an exotic quantum number hybrid. Based on these results in the charmonium sector, photoproduction appears to be a good place to look for hybrid mesons. In particular, the photoproduction of scalar mesons at intermediate energies could provide an alternative to the direct observation of the radiative decays. From the experimental point of view, the GlueX experiment [19] is being installed in the Thomas Jefferson National Accelerator Facility (JLab) accelerator. Its primary purpose is to understand the 
nature of confinement in QCD by mapping the spectrum of exotic mesons generated by the excitation of the gluonic field binding the quarks. The experiment will be able to probe new areas using photoproduction to produce exotic states.

Here, we focus on photoproduction of mesons states $a_{0}(980), f_{0}(1500)$, and $f_{0}(1710)$. The $f_{0}(1500)$ and $f_{0}(1710)$ mesons will be considered distinct in distinct mixing possibilities, assuming that $a_{0}(980)$ is member of the ground-state nonet. This is an established idea, although the motivation is different from that in some other works in the literature (e.g., Ref. [20]). The theoretical formalism employed is the Regge approach with Reggeized $\rho$ and $\omega$ exchange [17]. This paper is organized as follows: in next section we present the main expressions for scattering amplitudes and cross-sectional calculation of scalar meson photoproduction in the Regge theory, and in last section we show the numerical results and discuss distinct mixing scenarios and our main conclusions.

\section{MODEL AND CROSS-SECTIONAL CALCULATION}

The reaction proposed is $\gamma p \rightarrow p M$, where $M$ is one of the resonances $a_{0}(980), f_{0}(1500)$, or $f_{0}(1710)$. In practice, the meson $M$ will decay into two mesons. The contribution from vector mesons can be eliminated by considering only the all-neutral channels, that is, the $\pi^{0} \pi^{0}, \eta^{0} \eta^{0}$, and $4 \pi^{0}$ decays of the scalar-referred mesons. In the scope of Regge theory, the differential cross section in the narrow-width limit for a meson of mass $m_{S}$ is given by

$$
\frac{d \sigma}{d t}(\gamma p \rightarrow p M)=\frac{|\mathcal{M}(s, t)|^{2}}{64 \pi\left(s-m_{p}^{2}\right)^{2}},
$$

where $\mathcal{M}$ is the scattering amplitude for the process, $s$ and $t$ are usual Mandelstan variables, and $m_{p}$ is the proton mass. For the exchange of a single-vector meson (for instance, $\rho$ or $\omega$ ),

$$
\begin{aligned}
|\mathcal{M}(s, t)|^{2}= & -\frac{1}{2} \mathcal{A}^{2}(s, t)\left[s\left(t-t_{1}\right)\left(t-t_{2}\right)\right. \\
& \left.+\frac{1}{2} t\left(t^{2}-2\left(m_{S}^{2}+s\right) t+m_{S}^{4}\right)\right] \\
& -\mathcal{A}(s, t) \mathcal{B}(s, t) m_{p} s\left(t-t_{1}\right)\left(t-t_{2}\right) \\
& -\frac{1}{8} \mathcal{B}^{2}(s, t) s\left(4 m_{p}^{2}-t\right)\left(t-t_{1}\right)\left(t-t_{2}\right),
\end{aligned}
$$

where $t_{1}$ and $t_{2}$ are the kinematical boundaries

$$
\begin{aligned}
t_{1,2}= & \frac{1}{2 s}\left[-\left(m_{p}^{2}-s\right)^{2}+m_{S}^{2}\left(m_{p}^{2}+s\right)\right. \\
& \left. \pm\left(m_{p}^{2}-s\right) \sqrt{\left(m_{p}^{2}-s\right)^{2}-2 m_{s}^{2}\left(m_{p}^{2}+s\right)+m_{S}^{4}}\right]
\end{aligned}
$$

and where one uses the standard prescription for Reggeizing the Feynman propagators, assuming a linear Regge trajectory $\alpha_{V}(t)=\alpha_{V 0}+\alpha_{V}^{\prime} t$ for writing down the quantities $\mathcal{A}(s, t)$ and $\mathcal{B}(s, t)$ :

$$
\begin{aligned}
& \mathcal{A}(s, t)=g_{A}\left(\frac{s}{s_{0}}\right)^{\alpha_{V}(t)-1} \frac{\pi \alpha_{V}^{\prime}}{\sin \left[\pi \alpha_{V}(t)\right]} \frac{1-e^{-i \pi \alpha_{V}(t)}}{2 \Gamma\left[\alpha_{V}(t)\right]}, \\
& \mathcal{B}(s, t)=-\frac{g_{B}}{g_{A}} \mathcal{A}(s, t) .
\end{aligned}
$$

Equation (2) is different from that presented in Ref. [17], which contains a typographical error. However, we have verified that the numerical results in Ref. [17] are correct.
TABLE I. The widths, $\Gamma(S \rightarrow \gamma V)$, for the radiative decays of the scalar mesons to vector mesons $V=\rho(\omega)$. They are presented in units of $\mathrm{keV}$.

\begin{tabular}{lcc}
\hline \hline Scenario & $f_{0}(1500) \rightarrow \gamma V$ & $f_{0}(1710) \rightarrow \gamma V$ \\
\hline 1 & $2519(280)$ & $42(4.7)$ \\
2 & $1458(162)$ & $94(10.4)$ \\
3 & $476(53)$ & $705(78)$ \\
\hline \hline
\end{tabular}

Here, nondegenerate $\rho$ and $\omega$ trajectories $\alpha_{V}(t)=\alpha_{V}(0)+$ $\alpha_{V}^{\prime} t$, with $\alpha_{V}(0)=0.55(0.44)$ and $\alpha_{V}^{\prime}=0.8(0.9)$ for $\rho(\omega)$, are assumed. In Eq. (4), one has that $g_{A}=g_{S}\left(g_{V}+2 m_{p} g_{T}\right)$ and $g_{B}=2 g_{S} g_{T}$. The quantities $g_{V}$ and $g_{T}$ are the $V N N$ vector and tensor couplings; $g_{S}$ is the $\gamma V N$ coupling. The $\omega N N$ couplings are rather well defined [21], and we have used $g_{V}^{\omega}=15$ and $g_{T}^{\omega}=0$ following Ref. [17]. The $\rho N N$ couplings are not well defined, and we have considered $g_{V}^{\rho}=3.4$ and $g_{T}^{\rho}=11 \mathrm{GeV}^{-1}$. The $S V \gamma$ coupling, $g_{S}$, can be obtained from the radiative decay width through [22]

$$
\Gamma(S \rightarrow \gamma V)=g_{S}^{2} \frac{m_{S}^{3}}{32 \pi}\left(1-\frac{m_{V}^{2}}{m_{S}^{2}}\right)^{3}
$$

In the case of the $f_{0}$ mesons being considered as mixed $n \bar{n}$, $s \bar{s}$, and glueball states, their radiative decays to a vector meson, $S \rightarrow V \gamma$, are expected to be highly sensitive to the degree of mixing between the quark-antiquark basis and the glueball [16]. Here, we consider three distinct mixing scenarios: (1) the bare glueball is lighter than the bare $n \bar{n}$ state; (2) the glueball mass is between the $n \bar{n}$ and $s \bar{s}$ bare states; and (3) the glueball mass is heavier than the bare $s \bar{s}$ state. For the meson $a_{0}(980)$, decay to $\rho$ assumes that it is a member of the ground-state nonet. The numerical values for the widths taking into account the effects of mixing on the radiative decays of the scalars on $\rho$ and $\omega$ (in units of $\mathrm{keV}$ ) are shown in Table I, and for $a_{0}(980)$ we have

$$
\Gamma\left[a_{0}(980) \rightarrow \gamma \rho(\omega)\right]=14(126) \mathrm{keV} .
$$

The widths considered are taken from Ref. [17]. Clearly, the width is largely model dependent and other approaches can be used. We call attention to the interesting work in Ref. [23], where the decays of a light scalar meson into a vector mesons and a photon $(S \rightarrow V \gamma)$ are evaluated in the tetraquark and quarkonium assignements of the scalar states. The different nature of them corresponds to distinct large- $N_{c}$ dominant interaction Lagrangians.

In what follows we present the numerical results for the scalar mesons considered in the present study and the consequences of the different mixing scenarios discussed above.

\section{RESULTS AND DISCUSSION}

Let us summarize the numerical results for the photoproduction of scalar mesons $a_{0}(980), f_{0}(1500)$, and $f_{0}(1710)$. The differential cross section for $a_{0}(980)$ is presented in Fig. 1 at $E_{\gamma}=9 \mathrm{GeV}$, which is vanishing in the forward direction due to the helicity flip at the photon-scalar vertex and has 


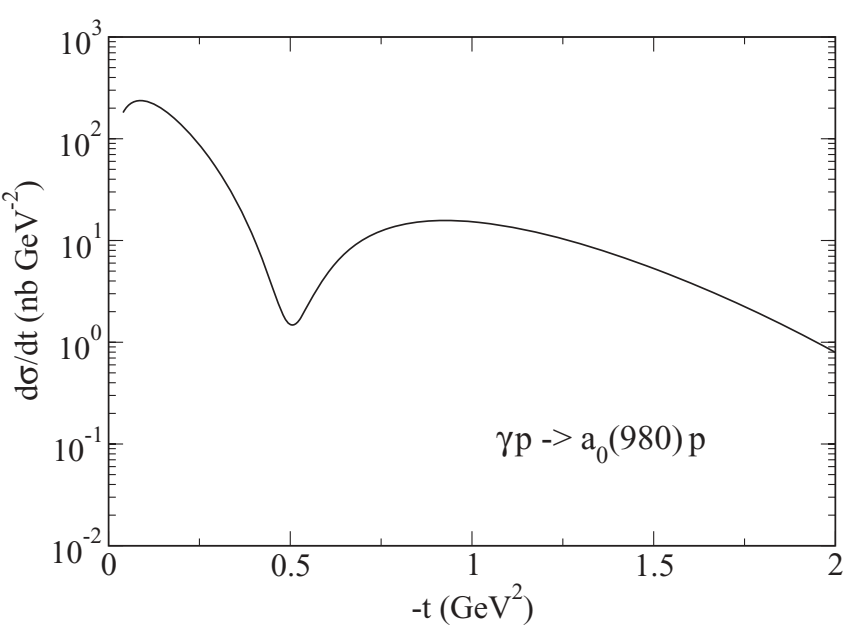

FIG. 1. Differential photoproduction cross section on proton for $a_{0}(980)$ at GlueX energy $E_{\gamma}=9 \mathrm{GeV}$.

a deep dip at $-t \approx 0.5 \mathrm{GeV}^{2}$. In the current scenario, the forward cross section is sizable, $d \sigma / d t_{t=0} \simeq 1 \mathrm{nb} / \mathrm{GeV}^{-1}$. The differential cross sections for $f_{0}(1500)$ are presented in Fig. 2 at $E_{\gamma}=9 \mathrm{GeV}$ and show the consequences of distinct mixing scenarios. The general structure follows the previous figure. In the scenario (I) the cross section is higher the other scenarios. That is, a light glueball mass implies a larger cross section for the $f_{0}(1500)$ mesons. On the other hand, the inverse situation occurs for the $f_{0}(1710)$ mesons, as shown in Fig. 3, where the large cross section comes from the heavy glueball mass component. The cross sections reflect directly the radiative decay widths as can be verified from simple inspection of Table I. It was advocated in Ref. [17] that the ratios of cross sections could give the "weight" of the glueball content. For completeness, the integrated cross sections for photoproduction of the scalars on protons at $E_{\gamma}=9 \mathrm{GeV}$ are given in Table II for light (1), medium (2), and

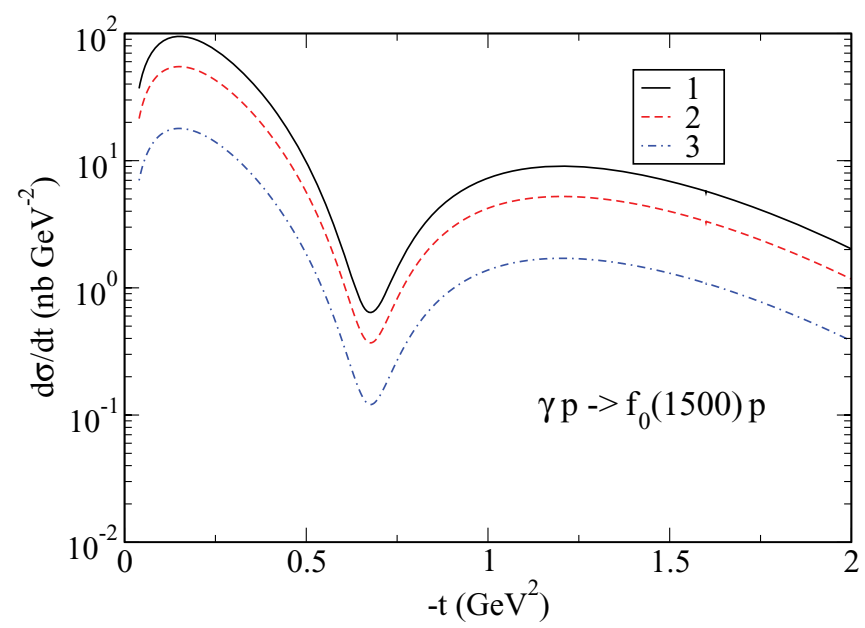

FIG. 2. (Color online) Differential photoproduction cross section on proton for $f_{0}(1500)$ at GlueX energy $E_{\gamma}=9 \mathrm{GeV}$. The results for the three distinct mixing scenarios are presented: 1 , solid line; 2 , dashed line; and 3, dot-dashed line.

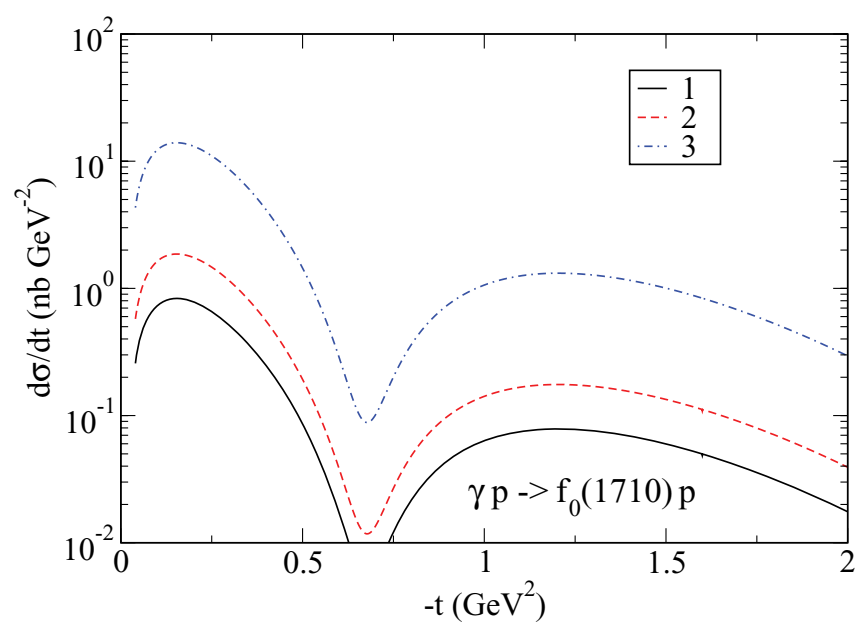

FIG. 3. (Color online) Differential photoproduction cross section on proton for $f_{0}(1710)$ at GlueX energy $E_{\gamma}=9 \mathrm{GeV}$. The results for the distinct three mixing scenarios are presented: 1 , solid line; 2 , dashed line; and 3, dot-dashed line.

heavy (3) glueball masses. For $a_{0}(980)$, the total cross section is $59.22 \mathrm{nb}$.

The background coming from the decay of vector mesons into two charged mesons can be eliminated by considering only the all-neutral channels, namely, the decays of the two neutral pions, two neutral $\eta$ 's, and four neutral pions of $a_{0}(980), f_{0}(1500)$, and $f_{0}(1710)$. An additional problem is the uncertainty in the branching fractions of $f_{0}(1710)$ [13] and its small cross section. On the other hand, the cross sections for photoproduction of the $f_{0}(1500)$ on protons are reasonable, and its branching fractions are much better defined. The scalars are not produced alone as in the $\pi^{0} \pi^{0}$ channel; there is a continuum background arising from the process of decay of photons in a neutral pion plus a vector meson like $\rho$ or $\omega$ with subsequent rescattering of the latter on the proton by $\rho(\omega)$ exchange to give the second $\pi^{0}$. This has to be taken into account in a realistic simulation of final-state configuration, as done in Ref. [17].

In summary, we have studied the photoproduction of the $a_{0}(980), f_{0}(1500)$, and $f_{0}(1710)$ resonances for photon energies relevant for the GlueX experiment at photon energy of $9 \mathrm{GeV}$. It would provide novel tests for our understanding of the nature of the scalar resonances and current ideas on glueball and $q \bar{q}$ mixing. The meson differential and integrated cross sections were evaluated, and the effect of distinct mixing scenarios were investigated. Although large backgrounds are expected, the signals could be visible by considering only the all-neutral channels, that is, their decays on $\pi^{0} \pi^{0}, \eta^{0} \eta^{0}$,

TABLE II. Integrated photoproduction cross sections in nanobarns on protons at $E_{\gamma}=9 \mathrm{GeV}$ for the three different mixing scenarios: light, 1; medium, 2; and heavy, 3. glueballs (see text).

\begin{tabular}{lrrr}
\hline \hline Scenario & \multicolumn{1}{c}{1} & \multicolumn{1}{c}{2} & 3 \\
\hline$f_{0}(1500)$ & 34.98 & 20.25 & 6.61 \\
$f_{0}(1710)$ & 0.30 & 0.68 & 5.08 \\
\hline \hline
\end{tabular}


and $4 \pi^{0}$. The theoretical uncertainties are still large, with $f_{0}(1500)$ the more optimistic case. Finally, an experiment in nuclei would also lead to the $f_{0}$ and $a_{0}$ excitation, mostly from the collision of protons with protons. The studies in nuclei would provide information on the meson properties in a nuclear medium, where large modifications are theoretically expected [24]. As a final note on the limitations of the present model, the narrow-width approximation for the scalars could be insufficient, taking into account that the final-state measured particles would be, for instance, $N \pi \pi, N K \bar{K}$, and $N \eta \pi$ and the energy-dependent width effects would become significant.

\section{ACKNOWLEDGMENTS}

The authors are grateful to A. Donnachie for useful remarks. We also thank Francesco Giacosa and Volker Crede for fruitful discussions. This research was supported by $\mathrm{CNPq}$ and FAPERGS, Brazil.
[1] N. A. Tornqvist and M. Roos, Phys. Rev. Lett. 76, 1575 (1996).

[2] N. N. Achasov, S. A. Devyanin, and G. N. Shestakov, Phys. Lett. B 96, 168 (1980).

[3] G. Janssen, B. C. Pearce, K. Holinde, and J. Speth, Phys. Rev. D 52, 2690 (1995).

[4] V. Mathieu, N. Kochelev and V. Vento, Int. J. Mod. Phys. E 18, 1 (2009); R. L. Jaffe, Phys. Rev. D 15, 267 (1977).

[5] P. Minkowski and W. Ochs, Eur. Phys. J. C 9, 283 (1999).

[6] W. Lee and D. Weingarten, Phys. Rev. D 61, 014015 (1999).

[7] F. E. Close and A. Kirk, Eur. Phys. J. C 21, 231 (2001).

[8] V. Crede and C. A. Meyer, Prog. Part. Nucl. Phys. 63, 74 (2009).

[9] G. S. Bali et al., Phys. Rev. D 62, 054503 (2000).

[10] F. E. Close and A. Kirk, Phys. Lett. B 483, 345 (2000).

[11] C. Amsler and F. E. Close, Phys. Lett. B 353, 385 (1995).

[12] E. Klempt and A. Zaitsev, Phys. Rep. 454, 1 (2007).

[13] K. Nakamura (Particle Data Group), J. Phys. G: Nucl. Part. Phys. 37, 075021 (2010).
[14] Y. Chen et al., Phys. Rev. D 73, 014516 (2006).

[15] K. Jansen, C. McNeile, C. Michael, and C. Urbach, Phys. Rev. D 80, 054510 (2009).

[16] F. E. Close, A. Donnachie, and Yu. S. Kalashnikova, Phys. Rev. D 67, 074031 (2003).

[17] A. Donnachie and Yu. S. Kalashnikova, Phys. Rev. C 78, 064603 (2008).

[18] J. J. Dudek, R. G. Edwards, and C. E. Thomas, Phys. Rev. D 79, 094504 (2009).

[19] Y. Van Haarlem et al., Nucl. Instrum. Methods A 622, 142 (2010).

[20] B. Kerbikov and F. Tabakin, Phys. Rev. C 62, 064601 (2000).

[21] R. Machleidt, K. Holinde, and Ch. Elster, Phys. Rep. 149, 1 (1987).

[22] Yu. S. Kalashnikova, A. Kudryavtsev, A. V. Nefediev, J. Haidenbauer, and C. Hanhart, Phys. Rev. C 73, 045203 (2006).

[23] F. Giacosa and G. Pagliara, Nucl. Phys. A 833, 138 (2010).

[24] E. Marco, E. Oset, and H. Toki, Phys. Rev. C 60, 015202 (1999). 